\title{
Nonviral Production of Human Interleukin-7 in Spodoptera Frugiperda Insect Cells as a Soluble Recombinant Protein
}

\author{
Maryam Mirzaei, ${ }^{1,2}$ Yan Xu, ${ }^{2}$ Cynthia B. Elias, ${ }^{2}$ and Satya Prakash ${ }^{1}$ \\ ${ }^{1}$ Biomedical Technology and Cell Therapy Research Laboratory, Department of Biomedical Engineering, and Artificial Cells and \\ Organs Research Centre, Faculty of Medicine, McGill University, 3775 University Street, Montreal, PQ, Canada H3A 2B4 \\ ${ }^{2}$ Biotechnology Research Institute (BRI), National Research Council (NRC), 6100 Royalmount Avenue, Montreal, \\ $P Q$, Canada H4P 2R2
}

Correspondence should be addressed to Satya Prakash, satya.prakash@mcgill.ca

Received 17 January 2008; Revised 14 May 2008; Accepted 7 August 2008

Recommended by Gerald Schumann

\begin{abstract}
Human interleukin-7 (hIL-7) is a cytokine secreted by the stromal cells of the red marrow. It is important for proliferation during certain stages of B-cell maturation and for T and NK cell survival, development, and homeostasis. It is a critical growth factor for enhancement and recovery of the immune T-cell. Because of its strong immunomodulatory effects, hIL-7 may become a valuable supplementary agent for immunotherapeutical treatments in patients with HIV infection or immunodeficiency. Human IL-7 has previously been produced in various protein expression systems. In this paper, we present an alternative expression system, in Spodoptera frugiperda cells, for the production of hIL-7 using nonlytic vector systems. This system allows generation of correctly translated and accurately processed heterologous proteins as soluble recombinant proteins. Here we report plasmid construction, transfection, and consequent expression of hIL-7 using this nonlytic insect cell expression system. The levels of secreted hIL-7 in a small scale experiment reached a level of $1.7 \mu \mathrm{g} \cdot 1^{-1}$ under serum-free cell culture conditions.
\end{abstract}

Copyright (C) 2009 Maryam Mirzaei et al. This is an open access article distributed under the Creative Commons Attribution License, which permits unrestricted use, distribution, and reproduction in any medium, provided the original work is properly cited.

\section{Introduction}

Recombinant proteins produced in insect cell systems are useful for fundamental research in cell and molecular biology. In addition, they are important for commercial production of reagents, therapeutics, and vaccines for agriculture and human health applications [1]. To produce recombinant protein in insect cells, baculovirus expression vector system (BEVS) is a suitable and widely used eukaryotic system [2-5] for high-level expression of heterogonous proteins. However, for production and purification of proteins, this system has a number of disadvantages including the transience of virus-based expression and the considerable effort required for scale-up and maintenance of virus stocks. In addition, viral proteases and cell lysates can cause degradation of the desired proteins and it is difficult to separate recombinant protein from recombinant virus particles $[2,6]$. In order to resolve these problems, an alternative approach using a nonlytic, virus-free expression system has been adopted [7,
8] that uses early baculovirus promoters in either transiently or stably transformed cells from Drosophila melanogaster, mosquito, as well as Spodoptera cells [8-11]. In contrast to baculovirus infected cells, stable insect cells are able to continuously produce soluble recombinant proteins, which facilitate protein purification [7] and the proteins are also properly modified. However, the rate of protein expression in stably transformed cells is often lower than that of a conventional baculoviral system.

In this study we used a nonlytic system to produce human IL-7 (hIL-7). Human IL-7 is a single-chain $25 \mathrm{kDa}$ protein first identified in bone marrow cultures through its pre-B cell growth factor properties; it was later described as a potent T-lymphocyte growth factor [12-14]. It is produced locally by intestinal epithelial and epithelial goblet cells and may serve as a regulatory factor for intestinal mucosal lymphocytes. IL-7 develops and activates lymphocytes; it also stimulates lymphopoiesis in lymphopenic mice $[15,16]$. These findings suggest a possible clinical application of IL-7 
for accelerating lymphoid reconstitution in lymphopenic patients. A number of preclinical studies have demonstrated possible functioning of IL-7 in antitumor clinical applications and gene therapy for metastatic diseases. IL-7 can also promote engraftment of stem cells in mice receiving bone marrow transplants, leading to a possible use of hIL-7 in patients receiving bone marrow or peripheral blood stem cell transplants [12].

To examine the expression and production of hIL-7 in a nonlytic, baculovirus-free expression system, we used a stably transfected insect cell system cotransfected with an expression vector containing a silk moth-Bombyx mori promoter and a resistance plasmid carrying a selectable marker puromycin gene $[7,17,18]$. For comparison purposes, we used another plasmid containing OpIE2 promoter for high-level, constitutive expression of the gene of interest containing a Zeocin resistance gene for selection of stable cell lines $[19,20]$. We also examined production of hIL-7 in Sf9 insect cells using BEVS.

\section{Materials and Methods}

2.1. Cells and Media. Spodoptera frugiperda, Sf9 cells (Invitrogen, Carlsbad, Calif, USA) were cultured in SF-900 II medium (Invitrogen, Carlsbad, Calif, USA) and incubated in a shaker incubator at a temperature of $27^{\circ} \mathrm{C}$ and $115 \mathrm{rpm}$. The cells were maintained by passaging 1 to 2 times weekly at an initial cell density of $4-5 \times 10^{5}$ cells $\cdot \mathrm{mL}^{-1}$. During this process, the total and viable cell densities and the cell size were measured using the automated Trypan blue exclusion method (Cedex, Innovatis, Bielfeld, Germany).

2.2. Plasmid. Nonlytic Triple Express Insect Expression System: pIE1/153A (V4) (Cytostore, Calgary, Alberta, Canada) and plasmid pBmA-pac (Cytostore, Calgary, Alberta, Canada) carrying a selectable marker puromycin gene were used. For comparison the basic vector pIZ/V5-His (Invitrogen, Carlsbad, Calif, USA) was also used.

2.3. Construction of Expression Vector. The hIL-7 gene was amplified by PCR from pORF9-hIL07 transfer vector (InvivoGen, Hornby, Ontario, Canada) using oligonucleotide primers: Forward: GCCTACCTGGGATCCGGTCA$A C$ and Reverse: TCATCAATGTATGCGGCCGCCTTATCATGTCGAG and Vent polymerase (New England BioLabs, Ipswich, Mass, USA). The PCR product was subcloned into the BamHII and NotI site of pIE1/153A (V4) vector. The recombinant plasmids containing hIL-7 cassette in frame were confirmed by restriction endonuclease digestion and DNA sequencing.

2.4. Cell Culture and Transient Transfection. Sf 9 cells were seeded into six-well plates at a density of $5 \times 10^{5}$ cells $\cdot \mathrm{mL}^{-1}$ ( $2 \mathrm{~mL}$ per well). Cells were cotransfected with the plasmids pIE1/153A.hIL-7 and pBmA-pac or pIZ/V5-His.hIL-7 using Cellfectin (Invitrogen, Carlsbad, Calif, USA). The mixture was incubated for 45 minutes then added to the cells and incubated for 5 hours at $27^{\circ} \mathrm{C}$. The medium was then replaced with fresh medium (SF900 II) and the cultures further incubated at $27^{\circ} \mathrm{C}$. One week later, transfected cells were transferred to a medium containing puromycin (Gibco $\mathrm{BRL}$ ) at a final concentration of $5 \mu \mathrm{g} \cdot \mathrm{mL}^{-1}$ in the case of pIE1/153A.hIL-7 (or $500 \mu \mathrm{g} \cdot \mathrm{mL}^{-1}$ Zeocin in the case of pIZ/V5-His.hIL-7 transfection) and incubated for 3 weeks. To allow selection of resistant cells, transfected cells were propagated for a further 2 weeks in Sf900II supplemented with appropriate antibiotics.

After this initial selection, cell cultures were maintained in media lacking any antibiotics. To establish clonal cell lines, the cells were harvested by gentle scraping, aliquots were transferred to 24-well plates and the plates were incubated for seven days at $27^{\circ} \mathrm{C}$. The media were changed every 2 days with fresh media supplemented with an appropriate antibiotic. Individual colonies were selected and transferred to 96-well plates using a micropipette. After 57 days, cells were amplified in Sf900II until sufficient cell concentrations were present for analysis. Polyclonal cell lines were maintained at $27^{\circ} \mathrm{C}$ in Sf 900 II. Using a micropipette, ten to twelve well-isolated antibiotic-resistant colonies from a 96-well plate were selected, and the hIL-7 expression levels from the supernatant of each stable polyclone were examined by Western blot analysis. Cells were maintained in antibiotic-free medium for 20 passages and the stability of the transfected cells was monitored using western blots analysis.

\subsection{Expression of Recombinant hIL-7 in Stably Transfected} Insect Cells. For the expression of recombinant proteins, a pool of stably transfected Sf9 cloned cells was grown in serum-free medium (Sf900-II) in shaker flasks. The cells were seeded at a density of $5 \times 10^{5}$ cells $\cdot \mathrm{mL}^{-1}$ and their growth was monitored by taking samples. The total and viable cell densities as well as cell size were measured (using the automated Trypan blue exclusion method, Cedex, Innovatis, Bielfeld, Germany). The experiment was performed in triplicate and samples were taken at regular intervals. The cell lines were frozen using freezing media (10\% FBS, 10\% DMSO, and 80\% Sf900 II) and stored in liquid nitrogen for further analysis.

2.6. Production of hIL-7 in Insect Cells Using BEVS. Plasmid construction, bacmid preparation, insect cell transfection, and baculovirus stock preparation have been described elsewhere [21]. For comparison assays, recombinant baculovirus containing human interleukin-7 (rbac/hIL-7) was used at a multiplicity of infection (MOI) between 5 to 0.5 to infect Sf9 cells. The cells were cultured in shaker flasks and were incubated at $27^{\circ} \mathrm{C}$ with agitation at $115 \mathrm{rpm}$. Samples were taken at regular intervals of $24,48,72$, and 96 hours post-infection (hpi). Total and viable cell densities and cell size were measured using the automated Trypan blue exclusion method (Cedex, Innovatis, Bielfeld, Germany) during the infection process. Cell cultures were separated into supernatant and pellets by centrifugation. 

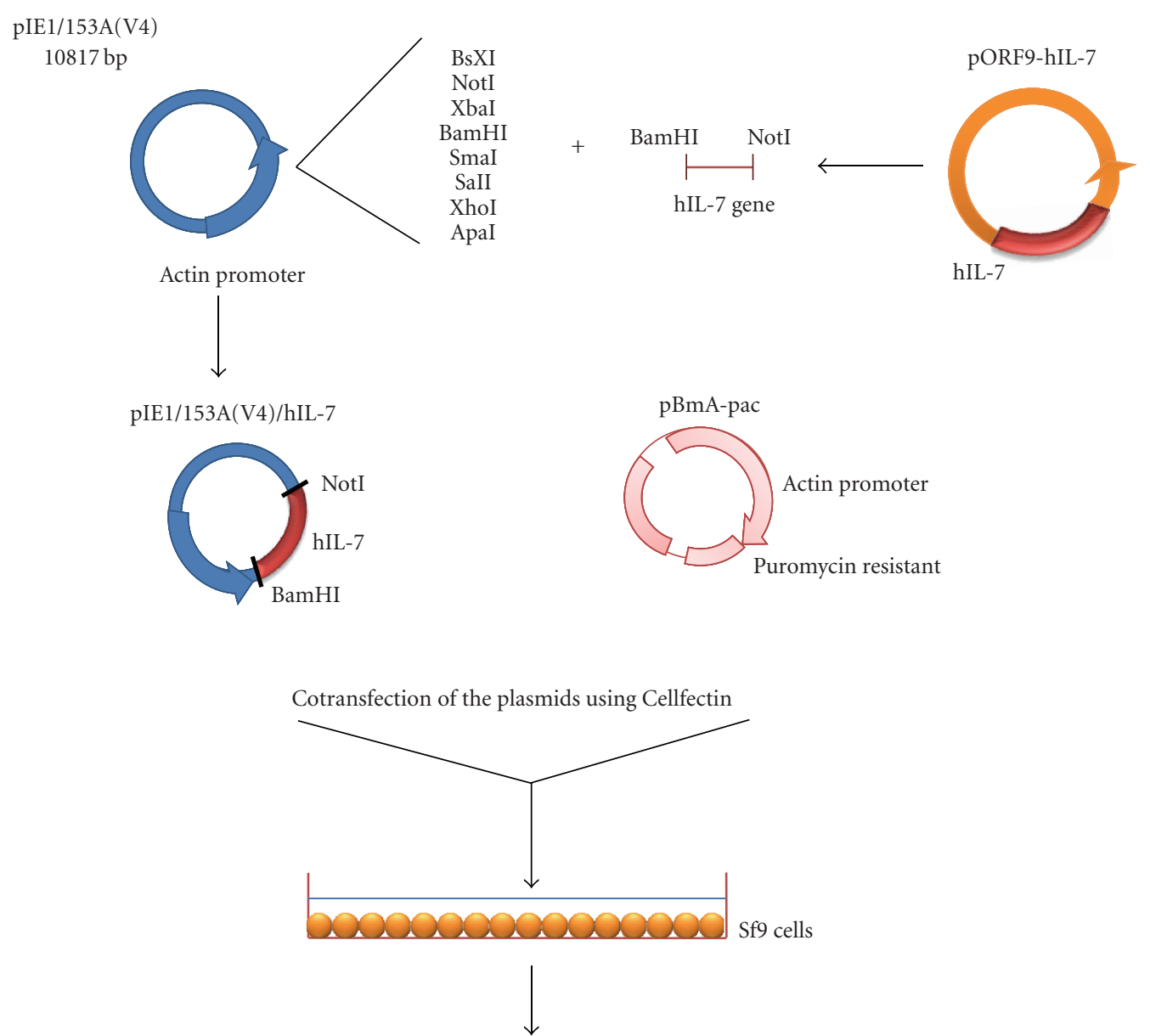

Selection of puromycin resistant clones

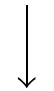

Analysis by Western blot and SDS page

FIgURE 1: Construction of the pIE1/153A (V4) expression plasmid carrying the hIL-7 gene. The hIL-7 gene from pORF9-hIL07 vector was inserted in pIE1/153A(V4) expression plasmid, pIE1/153A(V4)/hIL-7 and pBmA-pac were cotransfected into Sf9 cells. Clones producing hIL-7 were selected using puromycin and analyzed by western blot and SDS page.

2.7. Analytical Sssays. The amount of hIL-7 in supernatants was measured by sandwich ELISA using rabbit antihuman IL-7 antibody (RDI, Mass, USA) as the capture antibody and a biotinylated monoclonal antihuman IL-7 (R\&D systems, Ontario, Canada) as the detection antibody. Streptavidinhorseradish peroxidase (R\&D systems, Ontario, Canada) was used for conversion of a substrate mixture containing stabilized hydrogen peroxide and stabilized tetramethylbenzidine ( $R \& D$ systems, Ontario, Canada). The reactions were terminated by adding $\mathrm{H}_{2} \mathrm{SO}_{4}$, and the absorbance was measured at $450 \mathrm{~nm}$ using $570 \mathrm{~nm}$ as the reference wavelength. Commercially available purified human IL7(R\&D systems, Ontario, Canada) was used as a standard.

2.8. Western Blot Analysis. The expression of human interleukin-7 was determined using sodium dodecyl sulfatepolyacrylamide gel electrophoresis (SDS-PAGE). $15 \mu \mathrm{l}$ of samples were loaded to the gel and detected using a pol- yclonal rabbit antihuman IL-7 (RDI, Flanders, NJ, USA) as the primary antibody. The blots were developed using the chemiluminescence kit (Boehringer Mannheim, Mannheim, Germany) and visualized with the Kodak manager system.

2.9. hIL-7 Biological Sssay. Recombinant hIL-7 proteins produced in 2 different clones were tested for their bioactivity using induction of in vitro proliferation of an IL-7-dependent murine immature B lymphocyte cell line 2E8 (ATCC: TIB 239) as a measure of activity. 2E8 cells were maintained at $37^{\circ} \mathrm{C}$ in a $5 \% \mathrm{CO}_{2}$ atmosphere in Isocove's modified Dulbecco's medium (ATCC, Manassas, Va, USA) supplemented with $0.05 \mathrm{mM}$ 2-mercaptoethanol, $2 \mathrm{mM}$ glutamine, $50 \mathrm{IU} / \mathrm{mL}$ penicillin, $50 \mathrm{mg} / \mathrm{mL}$ streptomycin, $5 \mathrm{ng} / \mathrm{mL}$ IL-7, and 5\% fetal bovine serum (SigmaAldrich, Oakville, Ontario, Canada). IL-7 was found to remain biologically active in the presence of $0.05 \mathrm{mM}$ 2-mercaptoethanol. Cell proliferation was detected by 


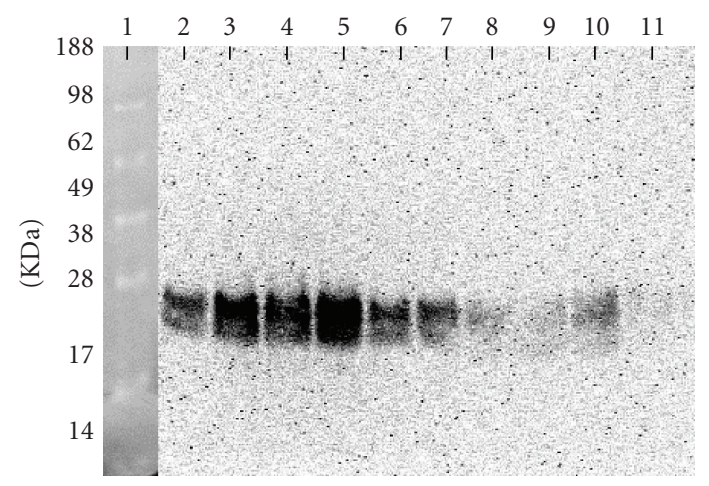

FIGURE 2: Screening of puromycin resistant clones in Sf9 cells cotransfected with pIE1/153A (V4) expression plasmid carrying hIL-7 gene and pBmA-pac carrying puromycin resistance gene. Cells were placed in medium containing puromycin at a final concentration of $5 \mu \mathrm{gmL}^{-1}$ for 3 weeks. Lane 1: protein marker. Lanes 2-11: polyclones Sf9hIL-7G, Sf9hIL-7E, Sf9hIL-7F, Sf9hIL-7A1, Sf9hIL7B4, Sf9hIL-7D5, Sf9hIL-7H, Sf9hIL-7C1-2, Sf9hIL-7C1-3, and Sf9hIL-B4-1, respectively.

tetrazolium compound [3-(4, 5-dimethylthiazol-2-yl)5-(3-carboxymethoxyphenyl)-2-(4-sulfophenyl)-2Htetrazolium, inner salt; MTS] and an electron coupling reagent (phenazine methosulfate: PMS) (Promega, Madison, Wis). For proliferation assays, 2E8 cells were washed three times and seeded at a density of $1 \times 10^{5}$ cells/well in 96-well plates in $50 \mu \mathrm{l}$ of medium containing no IL-7. A $50 \mu \mathrm{l}$ aliquot of crude protein was added to each well to give a range of concentrations from 0.01 to $100 \mathrm{ng} / \mathrm{mL}$ in a final volume of $100 \mu \mathrm{l}$. After 72-hours incubation, a $20-\mu \mathrm{l}$ aliquot of MTS-PMS (according to the manufacturer's protocol) was added to each well and incubated at $37^{\circ} \mathrm{C}$ for 4 hours. MTS is bioreduced by cells into a formazan product that is soluble in tissue culture medium. The absorbance of the formazan at $490 \mathrm{~nm}$ can be measured directly from 96-well assay plates without additional processing. Commercially available rhIL-7 preparations were used to establish the 2E8 Bioassay. Medium alone served as a negative control, and each sample was assayed in triplicate.

\section{Results and Discussion}

The hIL-7 gene was obtained by PCR reaction and digestion with NotI and BamHI enzymes from pORF9-hIL07 transfer vector, which were used to facilitate the insertion of the gene into the cut pIE1/153A (V4) or pIZ/V5-His expression vector plasmids. Cloned plasmids were then transferred to competent E.coli cells, purified according to the manufacturer's protocol, and used for transfection of Sf9 cells. Figure 1 illustrates the construction of pIE1/153A (V4) expression plasmid carrying hIL-7 gene (pIE1/153A.hIL-7) and the transfection procedure of the constructed plasmid into the Sf9 insect cells. For transfection, pIE1/153A.hIL7 plasmid was cotransfected with plasmid pBmA.pac containing puromycin resistance gene into the insect cells. The expression characteristics of the recombinant hIL-7 protein

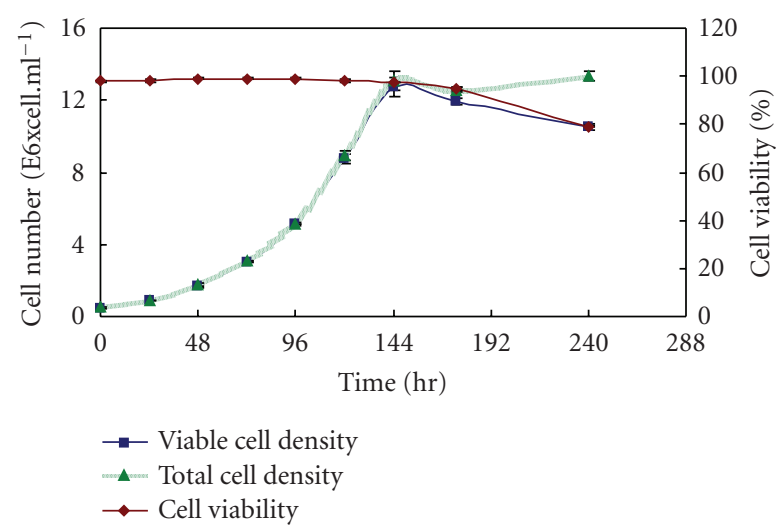

(a)

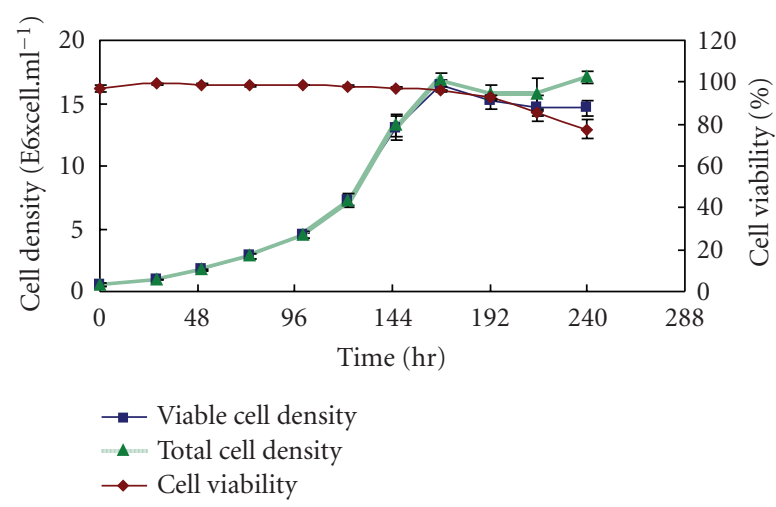

(b)

FIGURE 3: Growth curves of the stably transfected Sf9 producing hIL-7 and nontransfected Sf9 insect cells. (a) Viable cell density, total cell density, and viability of stably transfected Sf9 with pIE1/153A (V4)/hIL-7 plasmid. Cells were seeded at a density of $5 \times 10^{5}$ cells $\cdot \mathrm{mL}^{-1}$ and maintained at $1 \times 10^{7}$ cells $\cdot \mathrm{mL}^{-1}$. (b) Viable cell density, total cell density, and viability of nontransfected Sf9 insect cells. Cells were seeded at a density of $5 \times 10^{5}$ cells $\cdot \mathrm{mL}^{-1}$ $(n=3)$.

were analyzed by western blotting using hIL-7 specific antibody, as presented in Figure 2, which shows preliminary results for screening of positive clones. In Sf9 insect cells, production and processing of hIL-7 proceeded normally, resulting in a protein with molecular weight similar to that produced in mammalian cells. The most productive polyclone (Sf9hIL-7A1) was selected for further study.

Stable expression was verified by maintaining the Sf9hIL7A1 polyclone in puromycin-free medium after the selection. The expression of hIL-7 remained stable during more than 15 cell passages and cells could be maintained further in selection-free media without any decrease in their production. The cell lines were successfully frozen and retrieved from liquid nitrogen using the same procedures used for Sf9 cells. Figure 3 shows a growth curve over an 11-day period in Sf900 II for Sf9hIL-7A1 compared to nontransfected Sf9 insect cells. The cells appeared healthy and displayed an average doubling time of 24 hours. As shown in Figure 3(a), cell densities increased exponentially over the 


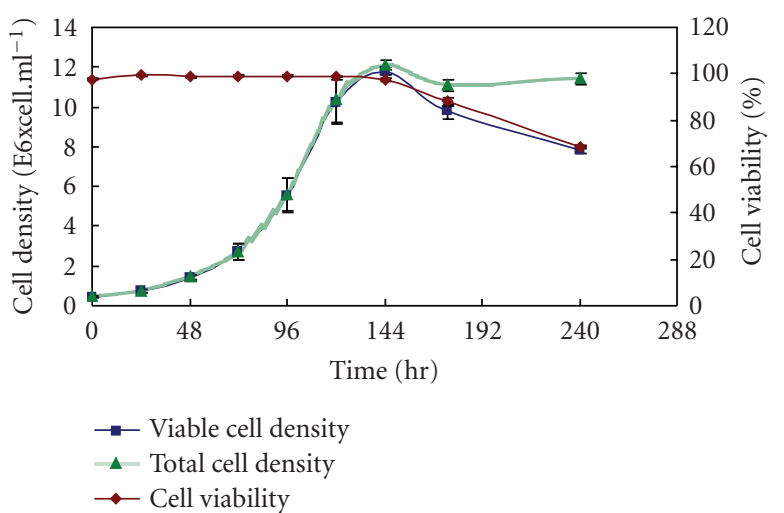

(a)

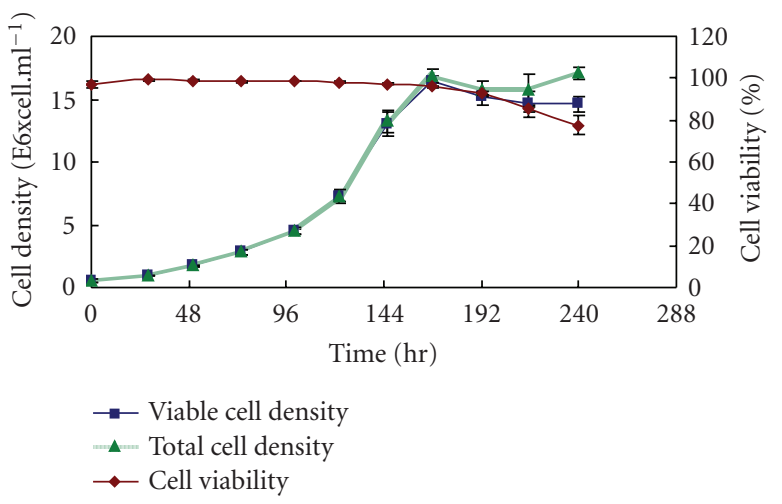

(b)

FIGURE 4: Growth curve of the stably transfected Sf9 producing hIL7 and nontransfected Sf9 insect cells. (a) Viable cell density, total cell density, and viability of stably transfected Sf9 with pIZ/V5-hIL7 plasmid. Cells were seeded at a density of $5 \times 10^{5}$ cells $\cdot \mathrm{mL}^{-1}$ and maintained at $1 \times 10^{7}$ cells $\cdot \mathrm{mL}^{-1}$. (b) Viable cell density, total cell density, and viability of nontransfected Sf9 insect cells. Cells were seeded at a density of $5 \times 10^{5}$ cells $\cdot \mathrm{mL}^{-1}(n=3)$.

first six days and then appeared to plateau between days six to eight, reaching maximum densities of approximately $8 \times 10^{6}$ cells $\cdot \mathrm{mL}^{-1}$. Culture viabilities remained high (above $95 \%$ ) until day seven but then dropped to below $70 \%$ by day eleven. Culture viabilities ranged from $95 \%$ to $99.5 \%$, with most determinations above $97 \%$ during the exponential stage, which was similar to nontransfected Sf9 insect cells (Figure 3(b)).

For comparison, we cloned the nucleotide sequences corresponding to the hIL-7 protein into the pIZ/V5-His expression vector and constructed the pIZ/V5-His.hIL-7 vector. This plasmid is an immediate early expression plasmid where the coding sequence for hIL-7 is positioned under the transcription control of the OpIE2 promoter. The promoter is derived from a second immediate early regulatory gene of the baculovirus Orgyia pseudotsugata multicapsid nuclear polyhedrosis virus (OpMNPV) which was identified in 1992 $[20,22]$. Insect cells were then transfected with $\mathrm{pIZ/V5-}$ His.hIL-7 and stable transfectants were selected with zeocin, a glycopeptide antibiotic of the bleomycin family, which

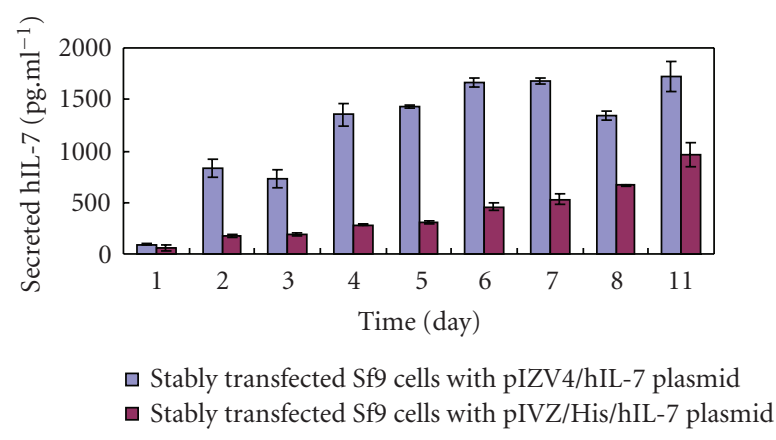

FIGURE 5: Production profile of hIL-7 in stably transfected Sf9 with two different expressions, cells were grown in serum-free medium (Sf900-II) in shaker flasks. The amount of hIL-7 in supernatants was measured by sandwich ELISA using rabbit antihuman IL-7 antibody as the capture antibody and a monoclonal antihuman IL-7 as the detection antibody. Streptavidin-horseradish peroxidase was used for conversion of a substrate mixture containing stabilized hydrogen peroxide, and stabilized tetramethylbenzidine commercially available as purified human IL-7 was used as a standard $(n=3)$.

is active in vivo against most bacteria (including E. coli), eukaryotic microorganisms (i.e., yeasts), plant cells, and animal cells [19]. The most productive polyclonals, grown with Sf900II medium in shake flasks at $27^{\circ} \mathrm{C}$ and $120 \mathrm{rpm}$ from an initial concentration of $5 \times 10^{5} \mathrm{cell} \cdot \mathrm{mL}^{-1}$, reached a maximum cell density of approximately $8 \times 10^{6} \mathrm{cell} / \mathrm{mL}$ on the fourth day of culture with a cell viability of $93 \%$ (Figure 4(a)). This demonstrated that stable transfection of pIZ/V5-His.hIL-7 into Sf9 cells did not cause significant changes in the growth of the transfected Sf9-hIL-7 cells nor in the cell viability, when compared to growth curves of wild-type Sf9 cells obtained under the same conditions (Figure 4(b)).

Secreted protein was measured in cell culture supernatants collected every 24 hours for 1-11 days (Figure 5) and its concentration was determined by ELISA. The quantity of hIL-7 in supernatants attained a maximum accumulation of $1.7 \mu \mathrm{g} \cdot 1^{-1}$ of cell culture supernatant. As shown in Figure 5, production of hIL-7 was generally still increasing in the supernatant on day 11 . In case of the cells transfected with pIZ/V5-His.hIL-7, production of the recombinant hIL-7 increased to a value of $1 \mu \mathrm{g} \cdot 1^{-1}$ on the eleventh day of culture.

Recombinant hIL-7 produced in both systems was assayed for biological activity by measuring the stimulation of IL-7-dependent 2E8 cell proliferation in vitro. Maximal stimulation of proliferation occurred in the presence of hIL-7 and no difference was seen in the ability of recombinant hIL7 to induce the proliferation of cell populations compared with that of the commercially available purified hIL-7. As shown in Figure 6, incubation with hIL-7 concentrations above $1 \mathrm{ng} / \mathrm{mL}$ resulted in an increase in the $A 490 \mathrm{~nm}$ of $2 \mathrm{E} 8$ cell cultures, with no activity seen at lower hIL-7 concentrations. The bioactivity of the rhIL-7 produced by both recombinant systems is very similar.

We compared productivity of hIL-7 in Sf9 cells using BEVS. To infect cells, different multiplicity of infection was 


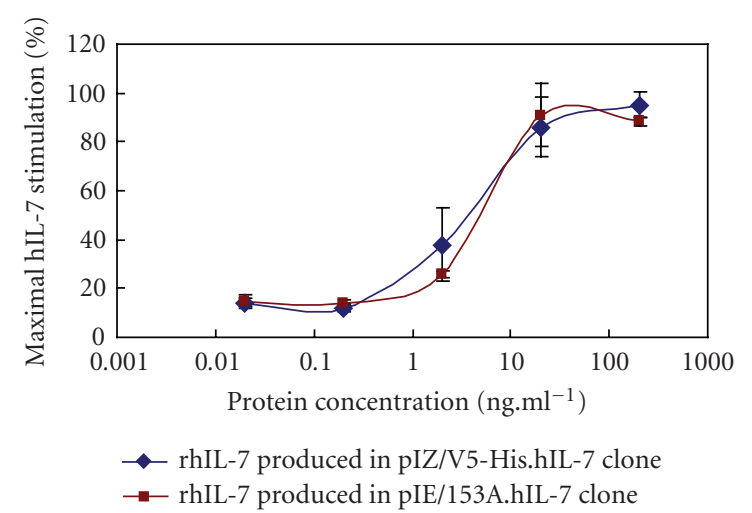

Figure 6: Biological activity assay of recombinant human IL7 produced in insect cells. IL-7-dependent murine immature B lymphocyte cell line 2E8 was used for proliferation assay. After 72 hours incubation at $37^{\circ} \mathrm{C}$ in a $5 \% \mathrm{CO}_{2}$ atmosphere, cell proliferation was detected by tetrazolium compound MTS and an electron coupling reagent PMS. The absorbance of the formazan was measured at $490 \mathrm{~nm}$. Commercially available rhIL-7 was used to establish the 2E8 bioassay. Medium alone served as a negative control, and each sample was assayed in triplicate $(n=3)$.

used. As shown in Figure 7, the final viable cell density of the uninfected cells (control) was much higher than those of cells infected by recombinant virus. The number of viable cells increased to more than $10 \times 10^{6}$ cells $/ \mathrm{mL}$ by 96 hours in the control and decreased to less than $1 \times 10^{6}$ cells $/ \mathrm{mL}$ in cells infected with the virus. In addition, the viability of infected cells decreased after $48 \mathrm{hpi}$, to less than $60 \%$. There was no significant difference in viability between control cells over a period of $96 \mathrm{hpi}$. The Western blot data shown in Figure 8 indicate that hIL-7 is produced and processed normally in the BEVS system to generate a protein with a similar molecular weight to that produced in mammalian cells. The protein was found in both the supernatant and the cell lysates, however, the intracellular protein was found to have multiple molecular weights. These different molecular forms are likely a result of incomplete glycosylation which may occur in this system.

In general BEVS produced higher amounts of recombinant hIL-7, as ELISA revealed that the expression level was almost 10 times greater in BEVS than in the stably transfected insect cells [21]. For production of hIL-7 in BEVS, a strong polyhedrin (PH) promoter for high-level protein expression was used. In this case, the baculovirus can synthesize recombinant protein in infected cells, polyhedrin eventually constitutes up to $50 \%$ of the total protein in the cell. This promoter has been used to construct expression vectors to allow high level of expression of the gene of interest under the influence of this promoter. In contrast, in the case of stably transfected insect cells, the silk moth-Bombyx mori promoter was used. This promoter is a region of the genome of Bombyx mori nuclear polyhedrosis virus (BmNPV) containing the homologous region 3 (HR3), which acts as an enhancer for the promoter of a nonviral gene, the cytoplasmic actin gene of the silkmoth. On the other hand, stable cultures, initiated with a lower cell density at

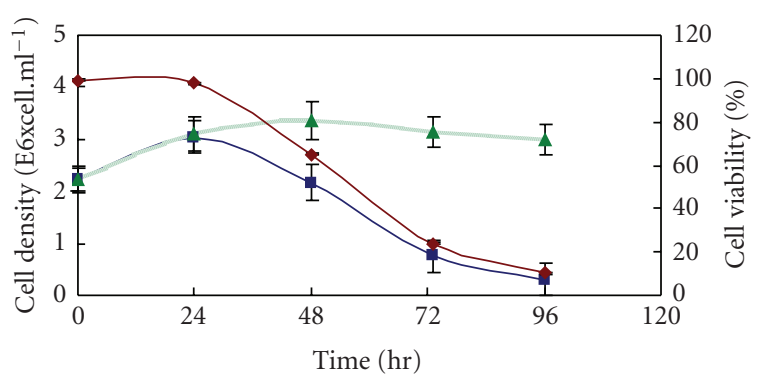

(a)

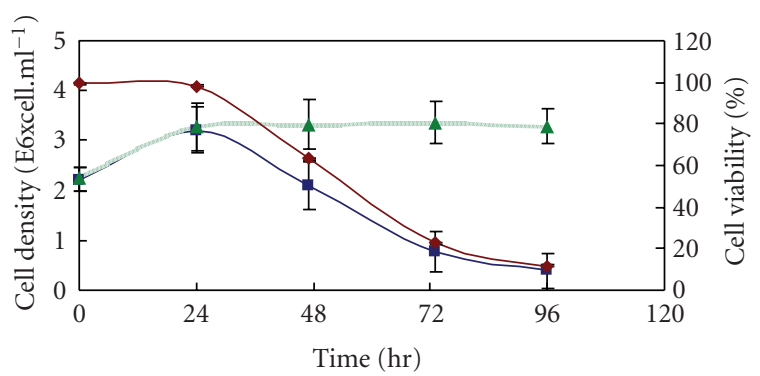

(b)

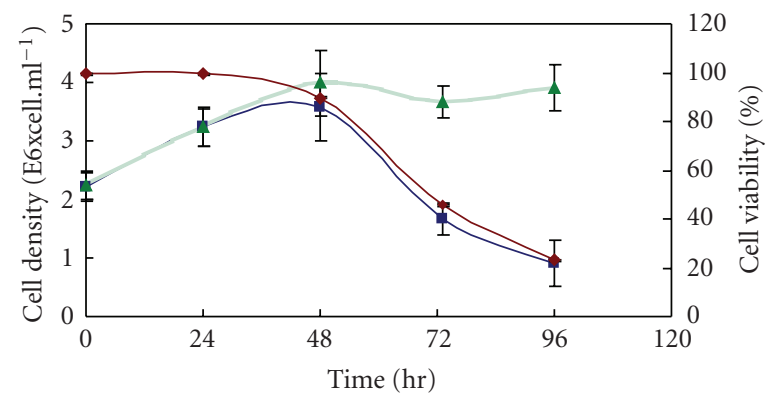

(c)

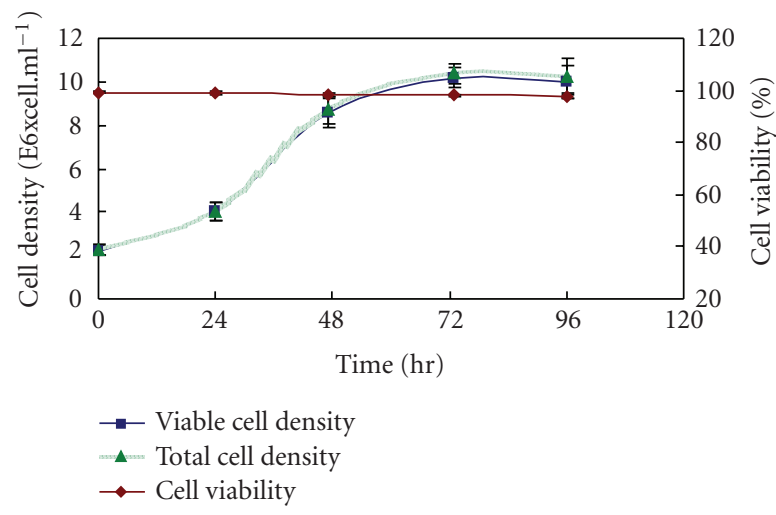

(d)

Figure 7: Viable cell density, total cell density and viability of the Sf9 insect cells infected with different MOI of rbacmid/hIL-7. Different multiplicity of infection (MOI) between 5 to 0.5 was used. Sf 9 cells were cultured in shaker flasks and incubated at $27^{\circ} \mathrm{C}$ with agitation at $115 \mathrm{rpm}$. Samples were taken at regular intervals $(24,48,72$, and 96) hpi. (a), (b), (c) Infected cells with MOI:5, 1 and 0.5 of the virus stock, respectively. (d) Control (Noninfected insect cells) $(n=3)$. 


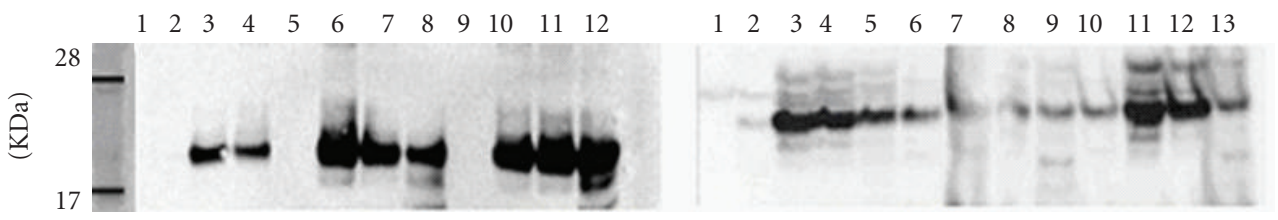

FIGURE 8: Western blot analysis of the intra- and extracellular of rhIL-7 produced in Sf9 insect cells. (a) Intracellular protein in Sf9: (lanes 13: 24 hpi, using MOI 5, 1 and 0.5 of the virus stock, respectively, lanes 4-6 48 hpi. Lanes 7-9: 72 hpi and lanes 10-12 96 hpi). (b) Extracellular protein in Sf9: (lanes: 2-4 24 hpi using MOI 5, 1 and 0.5 virus stock, respectively, lanes: 5-7 48 hpi, lanes: 8-10 72 hpi, lanes: 11-13 96 hpi).

$5 \times 10^{5}$ cell $/ \mathrm{mL}$, grew until they reached a maximal viable cell density of $13.3 \times 10^{6}$ cell $/ \mathrm{mL}$; whereas, the cells infected at $2 \times 10^{6} \mathrm{cell} / \mathrm{mL}$ cell density in case of BEVS reached a viable cell density of only $3.5 \times 10^{6}$ cell/mL. Viability of BEVS cells dropped to $10-20 \%$ at 96 hours post infection while in stably transfected insect cells, viability was maintained at 80-99\% for 240 hours incubation.

Generally, BEVS has been used for the high-level expression of eukaryotic genes in insect cells, as it is a rapid and convenient system for production of many different foreign proteins. The simplicity and rapidity of the vector system are two of its most important characters [2, 23], which make BEVS a powerful system for high-level, recombinant protein expression in insect cells. On the other hand, stable insect expression systems have advantages of both baculovirus and mammalian expression systems. They are also simple to use, with uncomplicated techniques for transfection and selection similar to those used with mammalian expression systems. Stable insect systems often reach higher levels of expression than do mammalian systems and are especially useful for production of secreted proteins [7].

In this study, we successfully established stably transfected insect cell lines for the production of recombinant hIL-7. We have demonstrated that these stably transfected cells can be used as an alternative to BEVS for large scale production of recombinant hIL-7. The levels of secreted human interleukin-7 in our small scale study reached $1.7 \mu \mathrm{g} \cdot \mathrm{l}^{-1}$ under serum-free cell culture conditions (in the case of the pIZ/V5-His.hIL-7 plasmid we obtained $1 \mu \mathrm{g} \cdot \mathrm{l}^{-1}$ ). In particular, the stable system using the expression vector pIE1/153A (V4) constitutes an advance for the expression of hIL-7 and possibly other glycoproteins in insect cells. This expression plasmid contains a silk moth-Bombyx mori promoter for high-level expression of the recombinant protein and a resistance plasmid which carries a selectable marker puromycin gene for selection of the transfected cells [7]. It was first constructed by Farrel et al. [7, 18] for continuous high-level expression of secreted glycoproteins by transformed lepidopteran insect cells and was developed as an alternative to baculovirus and mammalian cell expression systems. The expression cassette has the promoter of the silkmoth cytoplasmic actin gene, to drive expression of foreign gene sequences and also contains a transactivator gene and enhancer region of BmNPV, to stimulate gene expression. Since it is a nonlytic system, the glycoproteins obtained are probably more suitable for structural and functional studies.

\section{Acknowledgments}

This work was supported by research grants to S. Prakash from the Canadian Institute of Health Research (CIHR). The authors acknowledge Barbara Jardin, Rosa Tran, and Johnny Montes for technical assistance. M. Mirzaei acknowledges a Ph.D. scholarship from the Iranian Ministry of Health and Education.

\section{References}

[1] F. Altmann, E. Staudacher, I. B. H. Wilson, and L. März, "Insect cells as hosts for the expression of recombinant glycoproteins," Glycoconjugate Journal, vol. 16, no. 2, pp. 109123, 1999.

[2] L. K. Miller, "Baculoviruses as gene expression vectors," Annual Review of Microbiology, vol. 42, pp. 177-199, 1988.

[3] D. J. Fitzgerald, P. Berger, C. Schaffitzel, K. Yamada, T. J. Richmond, and I. Berger, "Protein complex expression by using multigene baculoviral vectors," Nature Methods, vol. 3, no. 12, pp. 1021-1032, 2006.

[4] E. Honjo, Y. Shoyama, T. Tamada, et al., "Expression of the extracellular region of the human interleukin-4 receptor $\alpha$ chain and interleukin-13 receptor $\alpha 1$ chain by a silkwormbaculovirus system," Protein Expression and Purification, vol. 60, no. 1, pp. 25-30, 2008.

[5] H. P. Lesch, S. Turpeinen, E. A. Niskanen, A. J. Mähönen, K. J. Airenne, and S. Ylä-Herttuala, "Generation of lentivirus vectors using recombinant baculoviruses," Gene Therapy, vol. 15, no. 18, pp. 1280-1286, 2008.

[6] M. D. Summers, "Milestones leading to the genetic engineering of baculoviruses as expression vector systems and viral pesticides," Advances in Virus Research, vol. 68, pp. 3-73, 2006.

[7] P. J. Farrell, M. Lu, J. Prevost, C. Brown, L. Behie, and K. Iatrou, "High-level expression of secreted glycoproteins in transformed lepidopteran insect cells using a novel expression vector," Biotechnology and Bioengineering, vol. 60, no. 6, pp. 656-663, 1998.

[8] M. J. Fraser, "Expression of eucaryotic genes in insect cell cultures," In Vitro Cellular \& Developmental Biology-Plant, vol. 25, no. 3, pp. 225-235, 1989.

[9] J. Benting, S. Lecat, D. Zacchetti, and K. Simons, "Protein expression in Drosophila Schneider cells," Analytical Biochemistry, vol. 278, no. 1, pp. 59-68, 2000.

[10] A. R. Bernard, T. A. Kost, L. Overton, et al., "Recombinant protein expression in a Drosophila cell line: comparison with the baculovirus system," Cytotechnology, vol. 15, no. 1-3, pp. 139-144, 1994. 
[11] T. A. Pfeifer, "Expression of heterologous proteins in stable insect cell culture," Current Opinion in Biotechnology, vol. 9, no. 5, pp. 518-521, 1998.

[12] Ö. Alpdogan and M. R. M. van den Brink, "IL-7 and IL15: therapeutic cytokines for immunodeficiency," Trends in Immunology, vol. 26, no. 1, pp. 56-64, 2005.

[13] R. G. Goodwin, S. Lupton, A. Schmierer, et al., "Human interleukin 7: molecular cloning and growth factor activity on human and murine B-lineage cells," Proceedings of the National Academy of Sciences of the United States of America, vol. 86, no. 1, pp. 302-306, 1989.

[14] Q. Jiang, W. Q. Li, F. B. Aiello, et al., "Cell biology of IL-7, a key lymphotrophin," Cytokine \& Growth Factor Reviews, vol. 16, no. 4-5, pp. 513-533, 2005.

[15] P. A. Welch, A. E. Namen, R. G. Goodwin, R. Armitage, and M. D. Cooper, "Human IL-7: a novel T cell growth factor," The Journal of Immunology, vol. 143, no. 11, pp. 3562-3567, 1989.

[16] M. B. Widmer, P. J. Morrissey, R. G. Goodwin, et al., "Lymphopoiesis and IL-7," International Journal of Cell Cloning, vol. 8, supplement 1, pp. 168-170, 1990, discussion 171-172.

[17] P. J. Farrell, L. A. Behie, and K. Iatrou, "Secretion of cytoplasmic and nuclear proteins from animal cells using novel secretion modules," Proteins: Structure, Function and Genetics, vol. 41, no. 1, pp. 144-153, 2000.

[18] P. J. Farrell, L. A. Behie, and K. Iatrou, "Transformed lepidopteran insect cells: new sources of recombinant human tissue plasminogen activator," Biotechnology and Bioengineering, vol. 64, no. 4, pp. 426-433, 1999.

[19] T. A. Pfeifer, D. D. Hegedus, T. A. Grigliatti, and D. A. Theilmann, "Baculovirus immediate-early promotermediated expression of the Zeocin ${ }^{\mathrm{TM}}$ resistance gene for use as a dominant selectable marker in Dipteran and Lepidopteran insect cell lines," Gene, vol. 188, no. 2, pp. 183-190, 1997.

[20] D. A. Theilmann and S. Stewart, "Molecular analysis of the trans-activating IE-2 gene of Orgyia pseudotsugata multicapsid nuclear polyhedrosis virus," Virology, vol. 187, no. 1, pp. 8496, 1992.

[21] M. Mirzaei, B. Jardin, C. B. Elias, and S. Prakash, "Expression and production of human interleukin-7 in insect cells using baculovirus expression vector system (BEVS)," Applied Biochemistry and Biotechnology. In press.

[22] D. A. Theilmann and S. Stewart, "Tandemly repeated sequence at the $3^{\prime}$ end of the IE-2 gene of the baculovirus Orgyia pseudotsugata multicapsid nuclear polyhedrosis virus is an enhancer element," Virology, vol. 187, no. 1, pp. 97-106, 1992.

[23] L. K. Miller, "Baculoviruses for foreign gene expression in insect cells," Biotechnology, vol. 10, pp. 457-465, 1988. 


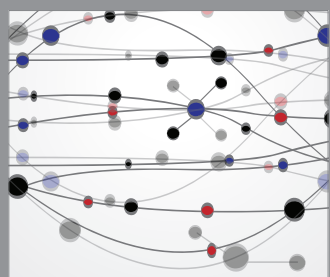

The Scientific World Journal
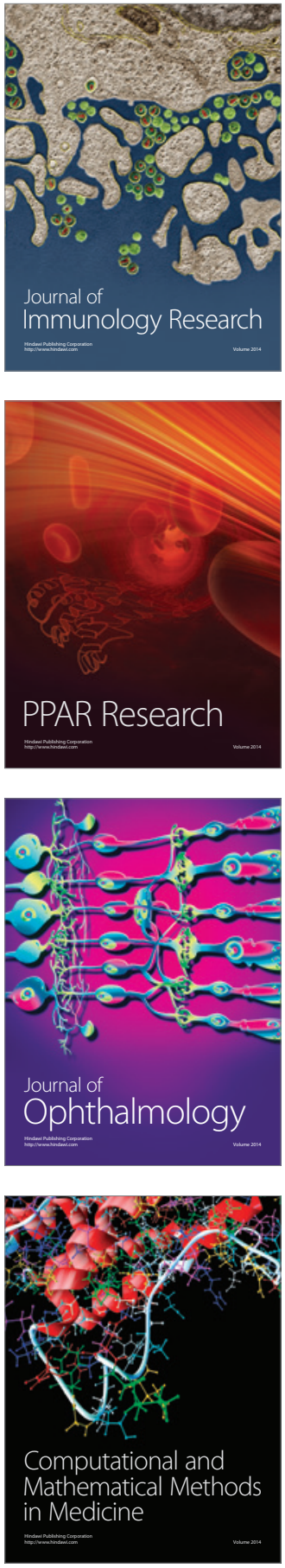

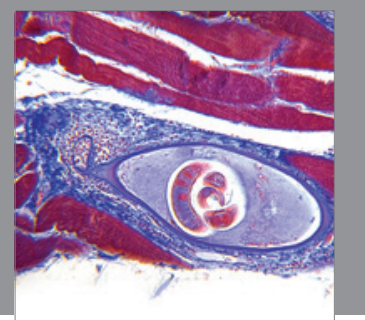

Gastroenterology

Research and Practice
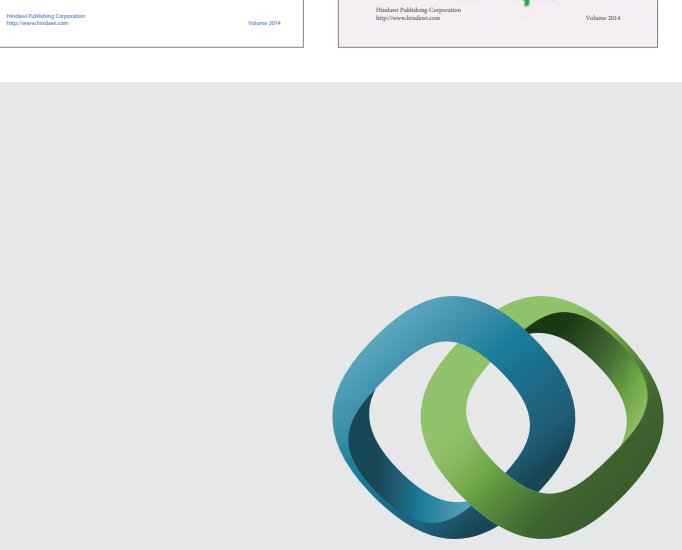

\section{Hindawi}

Submit your manuscripts at

http://www.hindawi.com
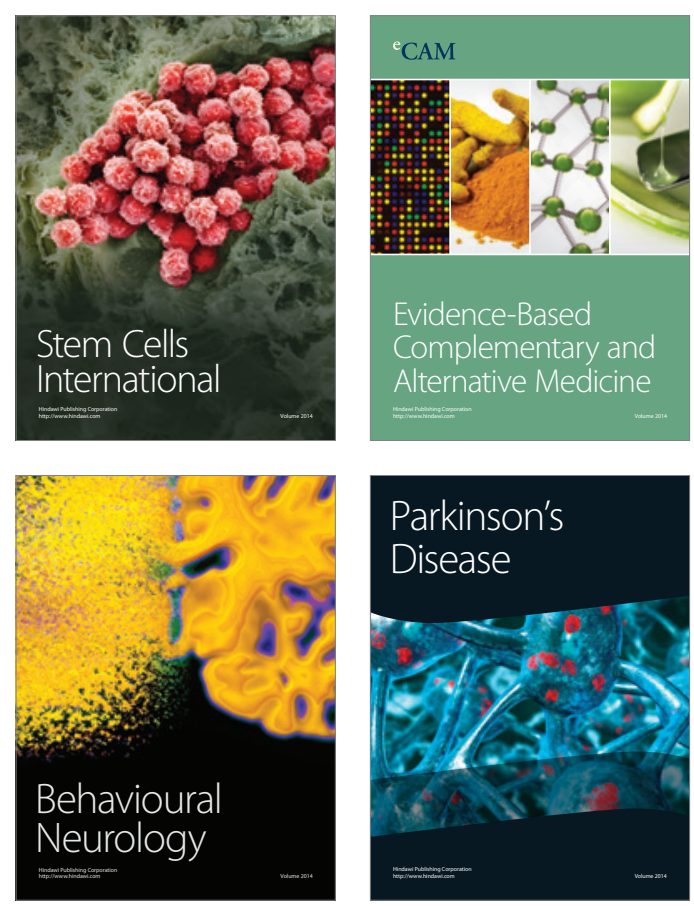

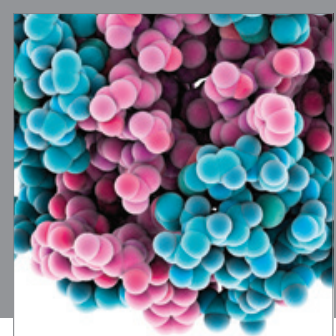

Journal of
Diabetes Research

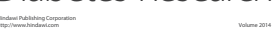

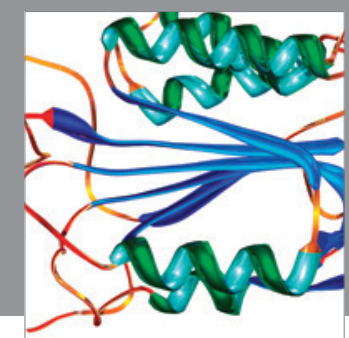

Disease Markers
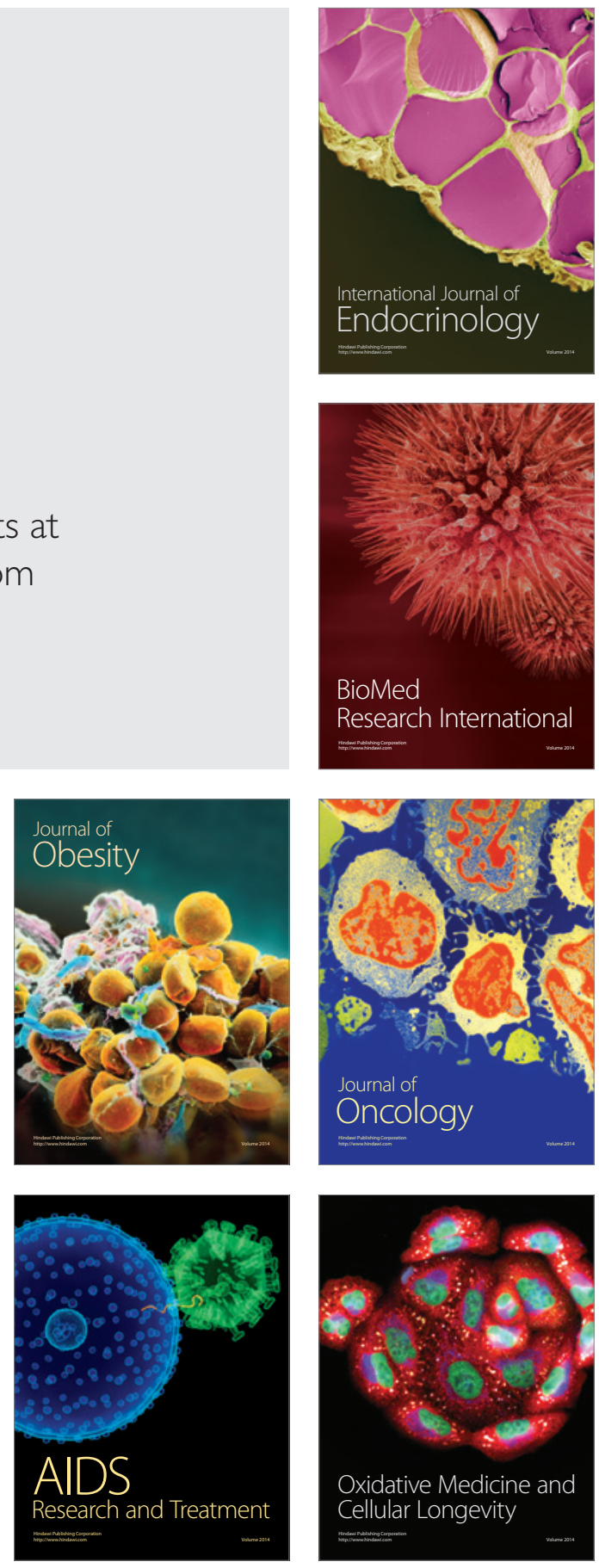